EOMmUn Communication et organisation

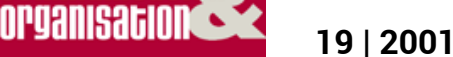

Actualité de la recherche en communication

\title{
Le théâtre d'entreprise : entre stratégie imaginee et stratageme imaginaire
}

Isabelle Cousserand

\section{OpenEdition}

12 Journals

Édition électronique

URL : http://journals.openedition.org/communicationorganisation/2527

DOI : 10.4000/communicationorganisation.2527

ISSN : $1775-3546$

Éditeur

Presses universitaires de Bordeaux

Édition imprimée

Date de publication : 1 mai 2001

ISSN : 1168-5549

Référence électronique

Isabelle Cousserand, "Le théâtre d'entreprise : entre stratégie imaginee et stratageme imaginaire », Communication et organisation [En ligne], 19 | 2001, mis en ligne le 27 mars 2012, consulté le 10 décembre 2020. URL : http://journals.openedition.org/communicationorganisation/2527 ; DOI : https://doi.org/10.4000/communicationorganisation.2527

Ce document a été généré automatiquement le 10 décembre 2020.

(c) Presses universitaires de Bordeaux 


\title{
Le théâtre d'entreprise : entre stratégie imaginee et stratageme imaginaire
}

\author{
Isabelle Cousserand
}

\section{NOTE DE L'AUTEUR}

Cet article s'appuie sur un mémoire de D.E.A. dont le corpus était constitué par près de 90 articles de presse (parus de 1993 à 1999), émanant de la presse généraliste, spécialisée, professionnelle et d'entreprise ; par un certain nombre de plaquettes et de documents recueillis auprès d'une dizaine de prestataires, prestataires que nous avons également rencontrés ou interrogés par téléphone. Enfin, nous avons complété cet ensemble par le témoignage de plusieurs commanditaires.

« L'expérience imaginaire de l'homme est plus vaste que son comportement social. »Jean

Duvignaud, Le don du rien, p. 300

1 Théâtre dans l'entreprise ? Théâtre par l'entreprise ? Théâtre sur l'entreprise ? Théâtre de l'entreprise... ? Disons-le d'emblée, théâtre d'entreprise est une expression ambiguë. Cette formule elliptique évoque communément du théâtre fait pour l'entreprise, voire par les acteurs de l'organisation. C'est une première précision que nous nous devons de moduler. Un rapide coup d'œil sur les annexes d'un ouvrage paru en 1998 et dénombrant moins d'une vingtaine de prestataires ${ }^{1}$, nous laisse supposer, non pas un théâtre d'entreprise, mais des formes théâtrales dans les organisations.

2 Le théâtre s'inscrit depuis la tragédie grecque (en relation avec la démocratie athénienne) dans une perspective morale et sociale. Il suffit de parcourir les ouvrages que Jacqueline de Romilly a consacrés à la tragédie.pour en être convaincu². Si le théâtre s'apparente à une manifestation artistique d'ordre social, il peut également être envisagé comme forme d'une expérience sociale qui revêt ainsi une dimension créative et spectaculaire. 
Spectacle théâtral ou théâtralisations sociales, la sociologie tient compte de ces deux aspects. "À tous les niveaux de la vie sociale, la cérémonie ou le spectacle constitue l'expérience collective et lui donne un sens en orientant les émotions ou les sentiments des groupes et des individus ». nous dit Jean Duvignaud ${ }^{3}$.

L'idée de faire venir des comédiens ou des artistes pour le compte d'une entreprise n'est pas nouvelle $e^{4}$. Il semble que l'utilisation du média ou du vecteur théâtre en communication organisationnelle ait d'abord prévalu sous les formes d'animations événementielles ou plus cérémonielles pour surprendre, dynamiser ou divertir, à la faveur d'opérations de relations publiques variées (conventions, anniversaires, assemblées générales...). Toutefois, son usage ne serait plus guère réductible à celui d'un «marketing événementiel $»^{5}$. Sa pratique se serait «internalisée » dès la fin des années 1980, plus volontairement associée aux projets managériaux. Ces formes d'interventions théâtralisées s'inscrivent alors autant dans les dimensions de la communication, du management, que de la formation. Elle viserait à valoriser, à sensibiliser, à prévenir, à motiver....

Le théâtre d'entreprise met ainsi en spectacle le travail et l'organisation. Il renvoie au vécu quotidien, met en scène les "symptômes " ${ }^{6}$, les tensions, les conflits, sous forme ludique, humoristique ou parodique. Ces interventions théâtralisées semblent revêtir principalement quatre formes ${ }^{7}$, dont les démarches diffèrent: la représentation d'une pièce ou de saynètes de catalogue transposables dans différentes organisations, la création d'une pièce ou de saynètes spécifiques au contexte d'une organisation, l'improvisation de comédiens ou d'artistes lors d'un événement (réunion, colloque, convention...), la représentation d'un spectacle qui peut aussi bien être donnée face au public d'une organisation ou face au "grand public». On peut en retenir une modalité particulière : la participation des acteurs de l'organisation (les salariés) à l'élaboration d'un scénario et à la représentation.

5 L'hétérogénéité de pratiques disséminées, leur faible degré de visibilité sociale et la multiplicité des acteurs concernés peut donc laisser à penser qu'il n'y a pas une image du " théâtre d'entreprise », mais bien une pluralité d'approches.

\section{Du théâtre dans les organisations}

\section{L'émergence d'une pratique}

6 Peu d'éléments sont à notre disposition pour cerner les origines du théâtre d'entreprise en France. Le premier ouvrage sur le sujet paraît en $1989^{8}$. Il s'agit d'un recueil de cinq pièces de théâtre en un acte sur la qualité, qui abordent des aspects managériaux liés au développement organisationnel et aux conduites comportementales. Les thèmes communicationnels interrogent différentes facettes des relations: la communication ascendante, la résolution, de conflit et la négociation, l'expression, l'écoute, le dialogue, les échanges commerciaux ou encore les paradoxes de la communication. En préambule, une note rappelle que les droits des pièces publiées ont été cédés à une société dont « (...) la vocation est d'apporter son concours à toutes les manifestations pédagogiques, telles que congrès, colloques, séminaires (...) ». Voilà un argument initial et incontournable du théâtre : sa dimension pédagogique. 
7 Selon les propos liminaires de l'auteur - propos intitulés « Le théâtre dans l'entreprise » tout commence par hasard vers le milieu des années 1980. lorsque l'un de ses amis entend l'une de ses pièces, écrite pour la radio et diffusée sur les ondes de France Inter. Création radiophonique, la pièce sera jouée dans le cadre d'un congrès sur la qualité, à la demande de cet ami. L'idée est lancée. On peut relever certains arguments véhiculés de nos jours sur le théâtre d'entreprise: les fonctions de miroir, de catharsis et de distanciation; le soutien en matière d'accompagnement au changement ou au développement; l'importance du vecteur de l'humour et du rire. Deux autres points sont abordés : la possibilité pour les comédiens de gagner un peu mieux leur vie et la contribution du théâtre à la « fabrication d'une culture », qu'il convient probablement d'entendre ici à travers les notions de-projet d'entreprise, de sentiment d'appartenance et de motivation'.

8 Michel Fustier apparaît comme l'une des figures pionnières ${ }^{10}$ du théâtre d'entreprise en France. Si certaines modalités d'intervention aujourd'hui rassemblées sous ce terme générique ont émergé à la même époque, ces formes théâtrales se sont diversement essaimées et de nouveaux prestataires ont développé une offre au contenu plurivoque.

\section{Une mosaïque d'interventions}

9 À compter de cette période, le théâtre d'entreprise évoque le plus souvent des pièces ou des saynètes, créées spécifiquement ou non pour une organisation; jouées par un petit nombre de comédiens (de un à cinq), sur un espace scénique plus ou moins restreint, de façon relativement dépouillée (peu d'accessoires et peu d'éléments de décor). Ces pièces ou ces saynètes portent généralement sur des thèmes managériaux qui relèvent fréquemment de l'actualité d'une organisation ou d'un phénomène actuel (la qualité, les 35 heures, l'euro, le bogue de l'an 2000...). Elles peuvent, selon le cas, revêtir de façon modulée des formes plus interactives. Elles sont souvent jouées face à un public interne, qui peut varier dans une fourchette de 10 à 2000 personnes. Les commanditaires sont plutôt des sociétés à capital conséquent et à effectifs importants. Toutefois, la palette des prestations vise à répondre aux attentes de tous types d'organisations.

10 Certaines représentations théâtrales peuvent recouvrir une dimension de spectacle plus avérée, car elles s'inscrivent dans un cadre festif: anniversaire ou inauguration, lancement de produit ou tout autre action susceptible d'être promue et célébrée en interne comme en externe... Ces spectacles relèvent autant de la création artistique que de la production événementielle ${ }^{11}$ véhiculent des enjeux, défendent des idées, valorisent une image...

11 On pourrait aussi mentionner les prestations des clowns. Leurs interventions peuvent recouvrir des formes variées, selon les objectifs assignés. Il peut s'agir de clownsanalystes ${ }^{12}$ qui se font connaître comme tels à l'orée des années 1980 , de clowns qui interviennent en milieu hospitalier auprès d'enfants malades et soumis à des protocoles médicaux lourds ${ }^{13}$, ou bien du clown d'un véritable cirque ${ }^{14}$ qui, dans le cadre d'un séminaire de management, intervient de façon ponctuelle pour surprendre et dérider les participants ${ }^{15}$. Citons également le one-man show ${ }^{16}$ qui est spécifique et peu décliné. Peu de prestataires se produisent seuls sur scène, qu'ils s'appuient sur le travail du clown, leur talent de comique ou même de mime, les personnages de la commedia dell $\operatorname{arte}^{17} .$.

12 À la marge, nous pouvons signaler des initiatives plus atypiques. Par exemple, une compagnie bordelaise propose des "petits théâtres à la carte » c'est-à-dire un théâtre «hors scène » qui s'inscrit dans une dimension de proximité. Des comédiens viennent 
jouer là où le public le souhaite, en investissant pour quelques minutes ou quelques heures des lieux familiers, professionnels ou insolites. D'autres peuvent organiser des visites théâtralisées de certains lieux touristiques...

Parmi les prestataires rencontrés ou interrogés, une majorité d'entre eux ont développé cette activité à l'orée des années 1990. Les structures qui sont antérieures sont soit à dimension artistique, soit cabinet de formation ou cabinet-conseil en management. Les années 1990 marquent donc un certain essor ${ }^{18}$. C'est notamment en 1991 qu'est créé le FITE. Festival international du théâtre d'entreprise, à Nantes.

Le lien qui unit le théâtre et l'entreprise n'est pas récent. Le théâtre, le spectacle sont venus dans les entreprises, à la faveur de manifestations diverses ou plus simplement par l'entremise de comités d'entreprise. Aujourd'hui un contexte très managérial semble porter ce théâtre. Mais il apparaît aussi que l'expression théâtre d'entreprise subsume plusieurs formes d'interventions (oserions-nous dire plusieurs genres ?). C'est un vocable " passe-partout » qui englobe différents prestataires et recouvre des registres distincts. Et parmi ceux que nous appelons prestataires, il convient également de citer ceux, minoritaires, qui se présentent en partenaires artistiques de l'entreprise. Par ailleurs, si le théâtre d'entreprise est souvent présenté comme un outil au service de la communication et de la formation, la formation semble prendre une part de plus en plus sensible ${ }^{19}$.

Une pluralité d'interprètes ${ }^{20}$ interviennent selon différentes modalités, ce que nous allons développer dans les lignes qui suivent.

\section{De « I'hyper-prose » à « Phyper-poésie »}

\section{Autour du principe de réalité : « prêt à jouer » et « sur mesure »}

Les prestataires du théâtre d'entreprise proposent des saynètes, des pièces ou des sketches écrits en fonction du contexte singulier d'une organisation. Cette représentation "sur mesure » est souvent destinée à un public interne. Dans une majorité des cas, le genre de ces spectacles est proche du café-théâtre, du show humoristique ou comique. On peut caractériser cette démarche en cinq, voire six étapes, dont les modalités peuvent différer selon le délai de création. Premièrement : contextualisation (rencontre, analyse des attentes et des besoins) et élaboration du cahier des charges. Deuxièmement : recueil des données (interviews, consultations de documents, observation plus ou moins participante...). Troisième étape : écriture du scénario, avec élaboration plus ou moins complète des textes selon les prestataires (la majorité fait valider les dialogues au mot près). Quatrième étape : mise en scène et répétitions, avec éventuellement filage en présence du commanditaire. Cinquième temps : représentation. Le sixième temps peut correspondre à celui d'un échange autour de ce qui vient d'être joué ou introduire un éventuel séminaire de formation. Le délai donné peut couvrir une période de quinze jours à deux mois ${ }^{21}$. La durée d'une pièce sur mesure est variable. Généralement, elle s'inscrit dans une fourchette de 20 minutes à une heure quinze minutes. La moyenne se situe autour de 30 à 35 minutes, d'un seul tenant ou en séquences ponctuelles. Plus elle est longue, plus elle nécessite de comédiens et de techniciens, plus clic revêt les formes $d$ un spectacle, plus son prix est exponentiel. Toutefois, les décors et les costumes sont souvent réduits au strict nécessaire (tenue de ville, table et chaises...). 
17 Dans le «sur-mesure » se dégagent deux grands axes de positionnement du théâtre. Le premier s'appuie sur un argument communicationnel. puisqu il s'agit de créer de meilleures conditions relationnelles, de renouer le dialogue, de valoriser certaines situations, en lançant une réunion, un débat, en dynamisant une convention, une inauguration ou en distrayant avec humour sur les questions abordées lors d'une grandmesse. En quelque sorte, le théâtre aide à mieux « faire circuler l'eau dans les tuyaux ». Le deuxième est d'ordre plus motivationnel. Il s'agit de créer les conditions d'une cohésion, d'impliquer, de rendre enthousiaste pour favoriser l'adhésion face aux changements ${ }^{22}$.

Venons-en au "prêt à jouer ». Il s'agit de traiter d'un thème généraliste touchant un grand nombre d'organisations. Les sujets abordés revêtent une dimension intemporelle (la délégation, l'accueil, l'entretien d'évaluation, la relation client-fournisseur...) ou sont inspirés par des questions d'actualité (l'euro, les 35 heures, l'an 2000...). Ces courtes pièces sont jouées face à un large public ou déclinées pour un public spécifique (l'euro pour les commerçants). Logiquement moins onéreux que le «sur-mesure » (notamment pour les PME/PMI ou des structures aux moyens plus modestes), il permet de répondre avec rapidité à certaines demandes et de rentabiliser une création. Selon les prestataires, la durée de ces représentations varie entre 15 et 35 minutes.

19 Le " prêt à jouer » permet plutôt de sensibiliser sur un sujet relevant de l'actualité d'une organisation. Ces courtes pièces ont fréquemment une fonction d'amorçage. La représentation permet ainsi de poser un problème généraliste tout en rendant le propos moins fastidieux.

\section{Sur le vif : réagir et improviser}

20 Autre registre d'interventions : les restitutions improvisées ou les réactions «à chaud ", en fonction du déroulement d'un événement. Peu de prestataires semblent proposer ce genre d'exercice qui demande une excellente capacité d'écoute, d'analyse, de synthèse et un travail d'interprétation singulier.

21 Il peut s'agir d'une "restitution sur le vif des moments forts d'un événement, $\mathrm{d}$ »'interventions flash" pour surprendre, "réveiller» l'attention ou effectuer une synthèse humoristique de certains moments. On peut également restituer la teneur des débats lors d'un séminaire de travail ou d'une réunion. Ces synthèses dépassent le niveau de ce qui se dit, pour s'attacher également aux attitudes des participants. Le but recherché est de créer une dynamique et aussi de mettre les publics face à leurs contradictions. Peu de prestataires mettent en avant une forme de dérision, d'ironie, de provocation $^{23}$. Cela renvoie à une dimension particulière de l'improvisation: celle du risque pris (peut-être balisé mais existant). On subvertit certains fonctionnements de l'organisation, on agit comme un bouffon ${ }^{24}$; ce qui implique que le commanditaire accepte les contraintes de ce mode d'intervention. Nous pouvons évoquer également le travail proposé par les clowns.

22 "Être clown est un état, une disposition à la poésie, plus qu'un métier »" ${ }^{25}$ La clownanalyse : l'art du clown ou le métier d'analyste? Les clowns interviennent à des moments jugés opportuns, par impromptus, qui s'apparentent à des ruptures d'ordre parodique. Un triple mouvement caractérise ces interactions : les réactions du public indépendamment de la présence du clown, l'interprétation du clown et enfin l'interrelation produite par la rencontre de ces deux "présences» et qui peut conditionner en partie ce que le clown va figurer au fil de son improvisation. C'est ainsi 
que se crée une forme de dialectique entre l'individu plongé dans une réalité dont il est partie prenante (ou qu'il subit) et le personnage imaginaire du clown. Toute intervention de clowns ou de comiques est par nature singulière, même si « quelques ficelles » peuvent être transposables d'un contexte à un autre. Ils ponctuent, mais ils font aussi contrepoint. Cette dimension de contrepoint peut s'expliquer ainsi : «A l'analyse institutionnelle, la clownanalyse (...) emprunte la conception d'une pratique de mise à jour des déterminations cachées des situations sociales et de leurs représentations fondatrices, bien au-delà de la rationalité des discours et des bonnes intentions. ${ }^{26}$. Les clowns improvisent en s'appuyant sur certains éléments des situations auxquelles ils peuvent assister de façon anonyme. Ils proposent une interprétation d'une réalité qu'ils observent dans un premier temps et dont ils deviennent initiateurs dans un second temps. Il s'agit ici de "dé-fonctionnaliser" c'est-à-dire de détourner des fonctions socialement instituées, de les subvertir. On peut isoler trois niveaux de détournement: le premier touche à ce qui se dit par exemple au propos d'intervenants; le deuxième relève de la façon dont le discours est reçu par les participants, notamment à travers leurs attitudes : le troisième se fonde sur les interactions entre les participants durant l'improvisation. On peut alors mettre en avant une autre dimension qui est celle du lien que tissent les clowns au fil de leur intervention. Les clowns proposent une approche autour de la dimension de "l'être ensemble». C'est une forme d'interrogation sur la nature des liens qui fondent l'individu et le groupe. Pour autant, tous les contextes n'y sont peut-être pas favorables. La puissance du clown vient de ce qu'il véhicule une dimension imaginaire. Comme le rappelle Jean Duvignaud, l'imaginaire «(...) est enraciné dans l'expérience réelle dont il est une partie composante : il fournit à celle-ci (...) des attitudes, des rôles, des situations sur lesquels les hommes projettent la même force que sur les actes religieux ou magiques. Ce sont des directions concrètes de l'expérience collective et individuelle, non des fantômes flottant dans le ciel des rêves ${ }^{27}$. Le travail du clown est très précis et en même temps très délié. Il suffit de très peu au clown pour déclencher l'hilarité. Le clown est drôle, le comédien aussi, mais il n'est pas un clown. " Il y a un don de soi chez un vrai clown, une ouverture vers l'autre, une fraîcheur, une naïveté, mais aussi une rébellion constante $»^{28}$.

\section{La parole contre le discours : interaction et participation}

Certains prestataires proposent de faire réagir le public en fonction de situations ou de faire jouer les salariés. Cette dimension participative du théâtre d'entreprise peut tout aussi bien être liée à des actions de formation, relever d'événements plus managériaux ou plus festifs, c'est selon. Faire jouer les salariés n'est pas anodin. Les arguments relevés sont souvent très motivationnels ${ }^{29}$. Selon le cas, ce sont soient les équipes sollicitées qui définissent les thèmes importants ou problématiques, soit le commanditaire. Ils peuvent aussi être suggérés par le prestataire. Les salariés participants sont généralement toujours impliqués dans l'écriture du spectacle ou des saynètes et sont encadrés par un ou plusieurs professionnels du spectacle. Certains mettent en avant que ces formes théâtrales servent autant des objectifs ludiques que stratégiques. D'autres assurent qu'elles «engendrent la motivation et l'implication", voire qu'elles "modifient les comportements ». Il s'agirait donc dans ce cas précis de faire admettre un certain nombre de règles comportementales. Ces interventions sont aussi envisagées comme un moyen pour «initier un processus de changement tout en renforçant l'esprit d'équipe et la 
cohésion ». On pourrait ici s'interroger sur les modalités de participation du public salarié et la valeur accordée à la parole, sous couvert de favoriser l'expression.

Autre forme déclinable : le théâtre ou le spectacle interactif. Le terme interactif recouvre en fait deux réalités distinctes. L'une s'inscrit plutôt dans une logique participante ludique ${ }^{30}$, l'autre est plus directement inspirée par la technique du «théâtre forum ». Le théâtre interactif inspiré par la technique du « théâtre forum », proposé par Augusto Boal dans le contexte singulier de la société (donc de la culture) brésilienne ${ }^{31}$, est l'apanage de rares prestataires. Ce théâtre est dit interactif car dans un deuxième temps du spectacle, "le spectateur [sollicité par un "meneur de jeu»32] a la possibilité d'arrêter le mouvement $d u$ spectacle sur une image pour améliorer et modifier le contenu directement devant le public $»^{33} \mathrm{Ce}$ deuxième temps dure de quinze à quarante minutes. Cette démarche permet d'opérer une sensibilisation et est une amorce pour nourrir la réflexion et le débat ${ }^{34}$. Les interventions des structures concernées se situent pour une large part dans les secteurs éducatif, culturel, sanitaire et social, autour des dimensions de la médiation sociale et de problématiques relevant par exemple du chômage, de la déviance, de la violence, des conduites dépendantes et à risque... Ces prestations peuvent être mandatées par des municipalités ou des associations pour intervenir auprès de publics fragilisés ou en situation de précarité. Ces techniques amènent ici des gens à raconter leur vécu, puis à le jouer et à le voir jouer par d'autres partenaires. Les histoires de chacun sont ainsi mises en scène et représentées devant un public auquel on donne la possibilité d'intervenir pour faire évoluer les situations représentées. L'extériorisation d'un vécu émaillé de difficultés et d'expériences douloureuses permet de retrouver une possibilité d'échange et de partage, à travers l'écoute des autres et de soi. Ces formes théâtrales permettent de démasquer et de réguler la violence des conflits. Elles ont une fonction d'exutoire, tout comme d'apprentissage et de régulation. Structurée autour d'une démarche rigoureuse, l'intervention offre la possibilité de créer une relation fondée sur la confiance et le respect. Ce travail théâtral contribue modestement à faire émerger l'expression d'une considération à travers l'échange de regards, de paroles, d'expériences et le partage d'un projet : le spectacle...

\section{Du principe de réalité au principe de plaisir : la création artistique}

Le spectacle, quelles que soient les formes qu'il requiert, sert souvent un ou plusieurs objectifs managériaux énoncés et négociés. Mais les formes théâtrales peuvent refléter une intention dictée par des enjeux organisationnels, relevant d'un «marketing événementiel " plus cérémoniel, et prenant les formes d'une production artistique. Il arrive ainsi qu'une compagnie théâtrale propose des créations tous publics. Le spectacle renvoie à une action de valorisation. Il singularise un événement, dynamise une inauguration, permet de «stabiliser» le public sur un site, tout comme l'attirer, le surprendre et le divertir. S'il ne sert pas nécessairement des objectifs cadrés, il peut recouvrir des intentions, sans pour autant que l'aspect artistique ne passe au second plan. À mi-chemin entre l'opération de relations publiques et l'invitation au spectacle, ce choix artistique s'appuie sur une combinatoire d'éléments qui visent à renforcer le capital image de l'entreprise aussi bien auprès de publics externes qu'internes. Il peut aussi permettre de se « démarquer » de la concurrence. Ce spectacle théâtral peut par exemple inciter à échanger sur un changement programmé, mais n'a pas pour argument de « faire changer $»^{35}$. Il peut s'agir de passer un moment convivial autour d'un sujet d'actualité 
(ex.: l'euro). Le théâtre peut ainsi s'inscrire dans le cadre d'un processus communicationnel très classique de relations publiques, où le fond sert la forme et vice versa.

Rappelons les grandes lignes qui semblent se dégager du vecteur de communication théâtral. Il revêt un caractère événementiel (de la réunion à la convention...). Il s'inscrit dans un moment précis, au sein d'un espace donné, en fonction d'une situation particulière et s'adresse à un collectif de personnes ${ }^{36}$. Comme pour toutes les opérations que nous avons évoquées, il prend d'autant plus de valeur si son exploitation est exceptionnelle (les commanditaires rencontrés s'accordent tous à le dire).

Dans les paragraphes suivants, nous revenons sur les dimensions communicationnelles des formes du théâtre d'entreprise et essayons de dégager en quoi le théâtre envisagé comme vecteur de communication peut véhiculer du sens ${ }^{37}$. Nous tentons de percevoir jusqu'où il participe du déferlement de «l'hyper-poésie " plutôt que de celui "l'hyperprose ». Comme le rappelle Edgar Morin: «Le déferlement de l'hyper-prose, c'est le déferlement d'un mode de vie monétarisé, chronométré, parcellarisé, compartimenté, atomisé, et pas seulement d'un mode de vie, mais aussi d'un mode de pensée où des experts spécialistes sont désormais compétents pour tous problèmes, et cette invasion de l'hyper-prose est liée au déferlement économico-techno-bureaucratique. Dans ces conditions, l'invasion de l'hyper-prose crée à mon avis la nécessité de l'hyper-poésie ${ }^{38}$.

\section{Mise en abyme de la communication}

\section{Équivoque du théâtre}

Le théâtre d'entreprise s'appuie sur une médiation humaine et interroge le cadre relationnel et la nature des relations qui construisent l'organisation. Il apparait souvent comme révélateur, vecteur de changement, de motivation. Ces dimensions ne se décrètent pas. Comme le rappelle Renaud Sainsaulieu : «L'homme n'est pas l'animal des béhavioristes et des pavloviens. La réponse à ses problèmes, aussi urgents soient-ils, s'inscrit dans un processus d'intervention, dans un milieu complexe de travail, dans une stratégie d'accès aux ressources, comme dans une interprétation par l'individu de la signification de ses propres récompenses et rétributions. ${ }^{39}$ Le théâtre peut sans doute accompagner le changement, mais il ne peut prétendre modifier les régulations sociales, car celles-ci reposent en partie sur les dimensions identitaires et culturelles. La culture d'une entreprise relève d'une production élaborée, fondée sur des identités, des valeurs et des représentations qui contribuent à tisser les relations ${ }^{40}$ au sein d'un collectif d'hommes et de femmes. Sommes-nous en présence d'une conception "émancipatrice » de la communication, telle que l'envisage par exemple Jean-Pierre Boutinet ${ }^{41}$ ? On pourrait avancer que plus le théâtre d'entreprise se veut porteur de messages significatifs, moins il devient porteur de sens, plus il perd de sa valeur communicationnelle, pour être assimilé à une technique managériale qui s'appuie sur un outil réputé communicationnel ${ }^{42}$. Comme le rappelle Patrick Baudry, « ce qui se retient au stade d'un sens voulu et maîtrisé n'atteint jamais le niveau proprement anthropologique d'un sens. $»^{43}$

On peut opérer une distinction entre un théâtre présenté comme outil susceptible de favoriser le dialogue, le débat ou la réflexion. en posant le problème sous forme humoristique et une action théâtrale présentée comme technique motivationnelle susceptible de favoriser l'adhésion. À travers l'idée d'une réflexion rendue possible par le 
théâtre (et son effet de miroir), les dimensions de débat et de dialogue sont quelquefois esquivées au profit de l'idée d'une prise de conscience, d'une transformation, qui amènerait le public au changement souhaité. La dimension de partage est quelquefois mise en avant, mais elle n'apparaît que faiblement. Toutefois, on peut relever l'idée de bienveillance et plus rarement d'empathie. La dimension d'expression se retrouve sous une forme plutôt larvée. On dit généralement que «le théâtre est plus efficace que les longs discours ", voire qu'il permet de "relancer la créativité »... L'idée de réassurance ${ }^{44}$ est présente. Face aux incertitudes, le théâtre permet de dédramatiser, de voir les choses sous un angle plus positif. "Dédramatiser » est repris comme un véritable leitmotiv et décliné sous divers aspects. L'idée du plaisir véhiculé par un spectacle est paradoxalement évoquée de façon très détournée. On s'attache plus aux effets produits. Parce que le spectacle, l'intervention théâtralisée fait rire, elle est plaisante... et également efficace. Enfin, on attribue à l'intervention comique des vertus cathartiques.

Ce que l'on nomme théâtre d'entreprise est donc une terre de contrastes qui entretient l'ambivalence entre un théâtre du réel (ou réaliste ?) et un théâtre de l'imaginaire ; entre un théâtre sans artifice (serait-il cependant artificiel?) ou dénué de tout parti pris esthétique et un théâtre qui se pose en terme de création. Là réside l'ambivalence de l'expression. Le discours oscille donc entre des arguments de type émancipateurs et de type aménageurs $^{45}$. Autour de l'aspect émancipateur, le théâtre s'apparente plus à un processus dont la finalité est communicationnelle: autour de l'aspect aménageur, le théâtre est plutôt une modalité de communication dont le dessein est plus motivationnel. Pour illustrer l'aspect aménageur du théâtre d'entreprise, on relève des expressions de type: "faire passer les messages", "faire passer quelques pilules", "médiatiser le changement ", " optimiser la réception des messages », "renforcer la communication", « vaincre les résistances », « recadrer », « aller chercher l'adhésion ». On joue sur l'aspect très classique du schéma émetteur-récepteur. On peut s'inscrire dans une dimension de persuasion, de mobilisation. Le théâtre est un «outil» (terme largement cité), un « levier ", un « déclencheur », voire un « alibi » qui procède d'une démarche managériale et sert une stratégie de communication motivationnelle. Pour ce qui est de l'aspect plus émancipateur, on relève des expressions du type: "informer", "faire remonter l'information", "questionner», "amorcer (ou renouer) le dialogue», "échanger ", «faire le lien (resserrer les liens)», « améliorer les relations ». Bref, des expressions qui ne témoignent pas seulement d'une communication descendante, mais ascendante et transversale, également orchestrale au sens où l'entend Yves Winkin: «(...) moins le contenu que le contexte, l'information que la signification $»^{46}$. Le théâtre, caractérisé encore comme un outil, devient plus un «mode» de communication, ou un «vecteur » (plus rarement cité) quelquefois une "aide». On pourrait donc qualifier le théâtre d'entreprise, soit d'un théâtre dans ou avec (aspect communicationnel - émancipateur), soit d'un théâtre pour ou par (aspect managérial - aménageur). Il ne s'agit pas de jouer l'un contre l'autre, mais cette distinction peut s'avérer utile pour saisir la nature des choix qui président à la venue de comédiens au sein d'une entreprise. L'argument avancé n'est pas artistique, esthétique ou culturel ${ }^{47}$, le théâtre est choisi pour motiver, pour susciter l'adhésion... l'idée d'un balancement entre logique fonctionnelle et instrumentale versus logique symbolique. 
Tableau 1 - Deux dimensions du théâtre dit d'entreprise

\begin{tabular}{c|c}
$\begin{array}{c}\text { Théâtre pour l'entreprise } \\
\text { Théâtre par l'entreprise }\end{array}$ & $\begin{array}{c}\text { Théâtre dans les organisations } \\
\text { Théâtre avec les organisations }\end{array}$ \\
\hline Aménageur & Emancipateur \\
\hline Contenu - Information & Contexte - Signification \\
\hline Objectif & Intention \\
\hline Produit & Action \\
\hline Technique de communication & Processus de communication \\
\hline Dimension fonctionnelle & Dimension instituante \\
\hline Management communicationnel & Communication \\
\hline Infléchir la motivation & Créer un climat \\
\hline Hétéronomie & Autonomie \\
\hline Faire passer les messages & Faire vivre un sentiment collectif
\end{tabular}

Il ne s'agit pas d'opposer chaque élément des deux colonnes, mais de caractériser chacune des démarches. Le fonctionnel peut être instituant, un processus se développer à travers une technique. Nous éclairons simplement certains aspects, identifiables dans les discours. Notre intention n'est pas de proposer une typologie, qui exclurait d'emblée des recoupements et signifierait que la réalité correspond au côté pile ou au côté face. Certains discours mêlent les deux dimensions, d'autres puisent de manière plus récurrente dans la première (pour - par).

L'idée de dynamisation de la vie organisationnelle n'est pas substituable au dynamisme de la vie de toute organisation. "Le dynamisme de la vie impose l'opposition, la différenciation et le conflit, mais il impose aussi simultanément, l'union, la solidarité et l'intercompréhension $»^{48}$.

\section{Arguments et invariants}

Comparativement à d'autres outils ou vecteurs de communication, le théâtre n'est pas démultipliable. Si l'on peut imprimer un journal en plusieurs exemplaires ou rediffuser un audiovisuel, la très grande majorité des interventions théâtralisées s'inscrivent dans une dimension d'actualité qui revêt incontournablement un caractère événementiel plutôt exceptionnel.

"Ludique et festif. lorsqu'il s'agit d'agrémenter une convention, le théâtre d'entreprise peut se révéler plus stratégique lorsqu'il s'agit de créer un climat précis ${ }^{49}$. Cette citation correspond à une définition plutôt récurrente du théâtre d'entreprise. Le théâtre apparaît bien souvent comme un alibi ludique pour dynamiser les salariés. Le plaisir éprouvé à assister à une représentation ou à un spectacle se dilue dans l'enthousiasme (qui est un effet recherché) ou dans une moindre mesure dans la convivialité véhiculée par les différentes formes d'interventions. Revenons en quelques lignes sur la question de l'effet miroir ou mimesis théâtrale, effet qui produirait la distanciation et favoriserait le changement. À travers la mimesis, on pourrait également suggérer que le théâtre d'entreprise pose la question de l'hétéronomie $»^{50}$, ce qui pourrait traduire un 
effacement des "significations imaginaires sociales ${ }^{51}$. Or la mimesis permet une mise en relation. Elle est liée au fait de «(...) se représenter moi et les autres, et d'interpréter le monde. $\|^{52}$ Autre credo du théâtre: dédramatiser. Il s'agirait de permettre de voir le problème autrement. L'idée de dédramatiser peut faire référence à une fonction de "réassurance contre l'angoisse» ou de "maîtrise du mouvant ${ }^{53}$. Toutefois, on peut y discerner aussi celle d'adhésion. Autour de l'argument d'un théâtre de réflexion et de changement, on relève l'idée d'un théâtre de pédagogie. Selon les circonstances, il s'agit de faire réfléchir ou de faire prendre conscience. On appréciera la nuance. Là encore tout dépend des intentions exprimées. On peut relever des détournements d'attitudes dans des situations professionnelles données ${ }^{54}$. Ou bien on peut sensibiliser des commerçants au passage à l'euro en proposant des saynètes qui mettront en évidence les adaptations nécessaires. Dans ces deux cas, la "prise de conscience" induite par la dimension pédagogique ne se situe pas au même niveau, même si les deux visent l'efficacité. Il s'agit de créer les conditions de l'action. Toutefois, on peut relever deux types d'arguments. Dans un cas. le théâtre sert à créer les conditions d'une réflexion pour faire naître le débat et instaurer le dialogue. Le miroir tendu par les comédiens devient ainsi la porte d'entrée à la discussion. Le théâtre permet de fédérer, de rassembler, d'unir les hommes. Cela peut aller de pair avec la recherche d'une cohésion. Mais cela peut aussi traduire le souci d'un sentiment collectif, qui peut aussi bien s'opérer dans une manifestation de type externe. Dernier argument que l'on retrouve fréquemment: le caractère innovant. Oserions-nous dire que c'est sans doute le cas de tous les outils de communication ou de management lorsqu'ils émergent? Ne disait-on pas il y a une quinzaine d'années que l'utilisation du vidéogramme d'entreprise était innovante? Comme nous l'avons déjà signalé, l'exploitation de spectacle accompagne depuis longtemps les événementiels. Les techniques de l'acteur servent régulièrement des actions de formation. La nouveauté réside en fait dans l'utilisation en interne. La démarche ne serait-elle pas plus innovante pour les comédiens qui sont amenés à jouer en entreprise et à gagner leur vie en partie de cette manière là, plutôt que pour l'entreprise elle-même ? Jusqu'où ce théâtre propose-t-il autre chose qu'une représentation qui a pour fonction de faire passer des messages? Sommes-nous face à une forme communicationnelle qui pourrait être en rupture avec l'idée de relation ${ }^{55}$ ? 
Tableau 2 - Petit lexique du théâtre d'entreprise

\begin{tabular}{c||c||c} 
Stratégique & Faire passer les messages & Effet de miroir \\
\hline Dédramatisant & Impliquer - Motiver & Humour - Rire \\
\hline Innovant & $\begin{array}{c}\text { Faire prendre conscience } \\
\text { Faire réfléchir }\end{array}$ & Changement \\
\hline Ludique & Fédérer - Rassembler & Efficacité \\
\hline Original & Dynamiser & Adhésion \\
& & Cohésion \\
& & Mobilisation \\
\hline Pédagogique & Sensibiliser & Effet cathartique \\
\hline & & Effet de surprise \\
\hline & & Enthousiasme \\
\hline & & Réflexion \\
\hline & & Convivialité
\end{tabular}

En caractère normal: adjectifs, verbes ou syntagmes verbaux, noms ou syntagmes nominaux les plus employés

En caractère italique: adjectifs, verbes, noms ou syntagmes nominaux fréquents mais moins employés

On pourrait se demander jusqu'où certaines formes du théatre d'entreprise ne chercheraient pas à infléchir la dynamique des jeux de pouvoir et jusqu'où l'aspect participatif (rendre acteur) ne viserait pas seulement le motivationnel, mais le comportemental, c'est-à-dire le normatif. Entre l'outil dit de réflexion et celui de changement et d'efficacité, il semblerait que les frontières soient poreuses. Certaines formes théâtrales n'apparaissent ni véritablement artistiques, ni véritablement engagées, mais souvent conformes aux idées que l'on souhaite véhiculer... Si le vecteur est présenté comme original, il n'incarne pas moins une certaine conformité. Rappelons qu'il se niche dans l'espace symbolique de l'autorité. Il pourrait s'avérer intéressant de discerner jusqu'où le théâtre d'entreprise ménagerait une combinatoire des règles dictées par la hiérarchie et de celles produites par les salariés; jusqu'où, en restituant le fonctionnement organisationnel. il rendrait compte des jeux des acteurs sociaux en présence ${ }^{56}$.

\section{En guise d'épilogue}

Le théâtre dit d'entreprise relève d'une médiation humaine et s'appuie sur une forme d'expression artistique. On peut donc être favorablement surpris et séduit par ce mode de communication ou d'intervention dans les organisations. On peut l'imaginer sensible et humain, pertinent et drôle, convivial et chaleureux. Et de surcroît, stratégique et efficace. Quel autre outil offrirait de tels attributs?

Les interventions théâtralisées revêtent des formes hétérogènes. Classiquement, elles visent à créer un climat relationnel favorable. Si les spectacles s'inscrivent depuis quelques années dans le cadre d'opérations de relations publiques, ce n'est que plus récemment 
qu'ils sont « utilisés » en interne. Nous pouvons constater qu'ils ne visent plus seulement à créer un climat, mais aussi à infléchir les motivations. C'est donc l'aspect participatif et motivationnel du management qui est souvent visé, à travers un vecteur qui s'appuie sur les dimensions de la sensibilité, du ressenti, des émotions. S'il apparaît convivial et s'inscrit quelquefois dans un cadre plus festif, le théâtre d'entreprise s'apparente souvent à une technique de gestion relationnelle à visée managériale. Jusqu'où l'utilisation de certaines formes théâtrales ne s'inscrirait-elle pas dans un contexte de rupture de la communication, c'est-à-dire de rupture des relations humaines et sociales qui fondent la vie organisationnelle?

Quoi qu'il en soit, il ne faudrait pas oublier que ce théâtre peut être source de bénéfices secondaires, tant du point de vue du public, que de celui des comédiens. Côté public, il permet de renouer avec le théâtre et de tisser un lien, même éphémère, avec les comédiens. Il peut également sensibiliser à l'expression théâtrale, voire créer des vocations en amateur. Côté comédiens, il permet de restaurer un contact avec le public et il assure à quelques uns de vivre décemment de ces formes de représentations, intérêt non négligeable pour une profession qui ne garantit pas toujours des moyens de subsistance à bon nombre des siens.

Unir le théâtre et l'entreprise peut offrir une échappée au gré des vents de l'imaginaire, mais il entrâne aussi dans le vent de la mode. Demandons-nous toujours au service de qui ces pratiques sont mises en œuvre, qui en lire profit et quelle en est la nature.

\section{BIBLIOGRAPHIE}

ARAGOU-DOURNON B., DETRIE P.. Le Théâtre d'entreprise : quand l'entreprise se met en scène. RueilMalmaison : Ed. Liaisons, 1998. 136 p. AUGE M., Pour une anthropologie des mondes contemporains. Paris : Flammarion. 1997, 195 p.

ASLAN O. (dir.). Le corps en jeu. Paris : CNRS Editions, 1993. 421 p. BALANDIER G.. Le détour : pouvoir et modernité, Paris : Fayard. 1985, 266 p.

BALANDIER G., Le dédale : pour en finir avec le XX siècle, Paris : Fayard. 1994, 236 p.

BAUDRY P., La place des morts : enjeux et rites, Paris : Armand Colin, 1999. 205 p.

BLANCHOT M.. L'espace littéraire, Paris : Gallimard, 1978 (lere éd. 1955), 382 p.

BOAL A., Jeux pour acteurs et non acteurs : pratique du théâtre de l'opprimé, Paris : éd. rev. et augni.. La Découverte, 1995, 260 p. BOAL A., Théâtre de l'opprimé, Paris : Ed. rev. et augm., La Découverte, 1996. 207 p.

BOUTINET J.-P., « Management par projet et logique communicationnelle : quelles convergences, quels défis? ", Communication \& Organisation : management par projet et logiques communicationnelles, $\mathrm{n}^{\circ}$ 13, GREC/O -Université Michel de Montaigne-Bordeaux 3, $1^{\mathrm{er}}$ semestre 1998, p. 207-221 CASTORIADIS C, Figures du pensable : les carrefours du labyrinthe VI, Paris : Editions du Seuil, 1999, $302 \mathrm{p}$. 
CHANLAT J-F., Sciences sociales et management : plaidoyer pour une anthropologie générale, ParisMontréal : Eska-Les Presses de l'université de Laval, 1998. 114 p.

CORVIN M., Dictionnaire encyclopédique du théâtre, Paris : Bordas, 1995 CROZIER M., FRIEDBERG E., L'acteur et le système, Paris : Editions du Seuil, 1992 (lère éd. 1977), 500 p.

DUVIGNAUD J., Les ombres collectives : sociologie du théâtre, Paris : PUF, 1973, 592 p.

DUVIGNAUD J., Le don du rien. Paris: Stock, 1977

FISCHER J-P.. PERRET J-B., « La mimesis sociale : l'approche historique de Gunter Gebauer et Christoph Wulf », Hermès : Mimesis, imiter, représenter, circuler, $\mathrm{n}^{\circ} 22$, Paris : CNRS Editions, 1998, p. 63-66

FUSTIER M.. L'entreprise mise en pièces... de théâtre : cinq comédies sur la qualité. Paris : Les Editions d'Organisation, 1989, 142 p. GUIRAUD M., « Bataclowneries vestimentaires », Pratiques corporelles $\mathrm{n}^{\circ}$ 110. mars 1996, p. 36-37

JEUDY H.-P., Les usages sociaux de l'art, Circé, 1999, 187 p.

LÉVINAS E.. Humanisme de l'autre homme, Paris : Le Livre de Poche, 1990

(lère éd. Fata Morgana 1972), 123 p.

MAISONNEUVE J., Les conduites rituelles, Paris : PUF, 1995 (lèreéd. 1988). 127 p.

MORIN E., Amour, poésie, sagesse, Paris, Editions du Seuil, 1999 (lère éd. 1997), 81 p.

ORGOGOZO I., Les paradoxes du management : des châteaux forts aux cloisons mobiles, Paris : Les Editions d'Organisation, 1991, 164 p. ROMILLY (de) J., La tragédie grecque, Paris : PUF, 1970, 192 p. ROUYER M-C, «Identité et identification dans quelques exemples du théâtre dans le théâtre : de la comédie classique emboîtée au one-man show ». Cahiers du Cert-Circe : Espaces du psychodrame et du théâtre, n 6, Bordeaux : Maison des Sciences de l'Homme d'Aquitaine, 1979, p. 69-98 SAINSAULIEU R., Sociologie de l'entreprise : organisation, culture et développement, Paris : Ed. rev. et augm.. Presse de la Fondation Nationale des Sciences Politiques \& Dalloz, 1997, 473 p.

SAUSSOIS J-M., « Coordonner, coopérer, adhérer : les enjeux du management », Sciences Humaines : comprendre les organisations, hors-série $n^{\circ} 20$, mars-avril 1998, p. 64-67

STOQUART J., Le marketing événementiel, Paris : La Découverte, Les Editions d'Organisation, 1991, $129 \mathrm{p}$.

WINKIN Y., Anthropologie de la communication : de la théorie au terrain. Bruxelles : De Boeck Université, 1996, 239 p.

\section{NOTES}

1. Béatrice Aragou-Dournon et Philippe Détrie, Le théâtre d'entreprise : quand l'entreprise se met en scène. Rueil-Malmaison, Ed. Liaisons, 1998

2. « (...) Il semble bien, en effet, que la tragédie n'ait pu naître que lorsque ces improvisations religieuses, d'où elle devait sortir, se trouvèrent prises en main et réorganisées par une autorité politique s'appuyant sur le peuple. (...) Entrée dans la vie athénienne par l'effet d'une décision officielle, s'insérant dans toute une politique d'expansion populaire, la tragédie apparaitt liée, dès ses débuts, à l'activité civique. ", J. de Romilly, La tragédie grecque, Paris, Puf, 1970, p. 13-15.

3. J. Duvignaud, Les ombres collectives : sociologie du théâtre. Paris, Puf. 1973. p. 105.

4. Les techniques théâtrales ont été diversement appliquées dans différents domaines: en psychologie, dans le champ éducatif, ou dans des démarches relevant de l'insertion (notamment 
par le biais du "théâtre-forum », quelquefois rebaptisé «théâtre interactif pour éviter tout anachronisme et se démarquer du contexte du « théâtre de l'opprimé » fondé par Augusto Boal).

5. Nous empruntons ce terme au litre d'un ouvrage de Jacques Stoquart. Le marketing événementiel. Paris, Les Editions d'Organisation, 1991

6. Nous nous référons à Ariane Mnouchkine qui dit à ce propos : " Le théâtre est l'art du « symptôme " ", Odette Asian, Le corps en jeu, Paris. Cnrs Editions, 1993, p. 296.

7. Nous ne développons pas précisément les approches relevant de la formation car hors cadre de cette étude. Mentionnons que les prestataires s'accordent incontestablement sur l'intérêt sensible (ou le regain d'intérêt ?) du théâtre comme outil de formation

8. Michel Fustier, L'entreprise mise en pièces... de théâtre, Paris, Les Editions d'Organisation. 1989. A cette époque. M. Fustier est consultant et dirige un cabinet-conseil en management.

9. « (...) rassembler les énergies de tous les membres de l'entreprise autour d'une «foi ». d'une conviction fondamentale, d'une passion commune (...) », M. Fustier. op. cit., p. 24.

10. B. Aragou-Dournon et Ph. Détrie. op. cit., p. 9.

11. On ne peut minorer la dimensio du spectacle ou spectaculaire.Interrogé dans le cadre de l'émission de

radio Agora (France Culture le 26.04.99),Jean Duvignaud disait « le possible es plus important que le réel ».

Comme le rappelle à un autre niveau Emmanuel Lévinas : «L'intériorité ne saurai tenir dans un dit »,E Lévinas Humanisme de l'autre homme. Paris le Livre de Poche. p 83

12. La « clownanalyse » a fait l'objet d'un dépôt de marque.

13. Par exemple l'association Nez à Nez, créée au début des années 1990.

14. Cirque Grüss

15. Nous faisons référence à une journée de formation organisée par France Télécom pour ses cadres commerciaux. Sans pour autant être l'élément majeur de cette journée, la présence du clown peut demeurer constante.

16. Le one-man show est un spectacle interprété par une seule personne, qui joue un ou plusieurs personnages.

17. Comédie italienne populaire, la commedia dell'arieest la comédie des masques. C'est un théâtre de création collective des acteurs qui improvisent gestuellement et verbalement à partir d'un canevas sommaire. L'improvisation prend ici en compte les réactions du public. La commedia dell'ariecompte une douzaine de figures qui se répartissent en deux familles : le parti grave et le patri ridicule qui porte toujours des masques (Pantalone. Pulcinella. Mezzotino. Scapino...).

18. Sur l'ensemble des 18 références répertoriées dans l'ouvrage de $\mathrm{B}$. Aragou-Dournon et $\mathrm{Ph}$. Détrie, onze affichent une date de création comprise entre 1990 et 1995, deux se situent dans la deuxième moitié des années 1980. cinq dans la première moitié (dont une à la lisière : 1979).Une minorité en vit de manière exclusive.

19. Reconnu comme outil de formation, il peut donc relever de ce budget.

20. Le fait d'interpréter concerne tout autant le processus de production que celui de réception. La signification s'élabore et se structure en amont comme en aval de la «transmission ». Si l'intention préside, l'interprétation couvre l'ensemble du processus. Le travail d'interprétation est créateur d'une relation qui permet de produire du sens: ce qui ne relève pas de la même chose que de faire passer un message. Nous pourrions revenir aussi à la tragédie qui «(...) se définit plus par la nature des questions qu'elle pose que par celle des réponses qu'elle fournit. ", J. de Romilly, op. cit., p. 167.

21. L'écart entre une forme de reproduction et une dimension de création devient alors plus ténu

22. Ce que l'on peut retrouver de façon plus ou moins nuancée autour d'expressions telles: "faire changer les comportements» ou «faire prendre conscience ». Dans une dimension plus 
communicationnelle. il sert des intentions plus générales: sensibiliser (à l'euro), rassembler autour d'une idée, favoriser le débat ou le dialogue (positionnement moins récurrent).

23. Ceci nous semble intéressant pour ce que l'on peut supposer du commanditaire et de ce qu'il convient de nommer la culture d'entreprise, car même si la représentation est censée fédérer et ne dévaloriser personne, l'humour manié n'est pas toujours consensuel.

24. Le personnage du bouffon ou du clown est celui du naif qui opère un travail de déconstruction et s'approprie tous les points de vue. Jean Duvignaud a mis en avant combien « (...) la fonction du bouffon est bénéfique pour le roi (...) puisqu'il a pour mission de réconforter la conscience du potentat, parfois chargé de remords et d'appréhensions graves. (...) En s'emparant des gestes, de la couronne et de l'allure du souverain (...) il fait le roi et les limites de son pouvoir sont celles que lui accorde le roi lui-même ». J. Duvignaud, op. cit.. p. 204-205.

25. Annie Fratellini. " Le corps du clown », in Odette Aslan, op. cit., p. 345-346.

26. Marc Guiraud. « Bataclowneries vestimentaires ». Pratiques corporelles $n^{\circ} 110$. mars 1996. p. 36-37.

27. J. Duvignaud. op. cit., p. 200.

28. A. Fratellini. op. cit.. p. 346.

29. On trouve des expressions du type: «optimiser leur rôle", "renforcer les énergies", "fédérer »... Il s'agit fréquemment de «dynamiser», ou de façon plus prosaïque de "faire changer ».

30. Un exemple parmi d'autres : dans le cadre d'un événement, d'un dîner..., les comédiens mettent en scène des situations truffées d'erreurs, en relation avec une thématique particulière. Il s'agit d'amener le public (quelquefois constitué en équipes pour la circonstance) à détecter ces erreurs et à proposer le correctif nécessaire.

31. A. Boal, metteur en scène et formateur brésilien, a proposé des formes d'interventions théâtrales « (...) fondées sur une idéologie révolutionnaire et un activisme politique » (Michel Corvin, Dictionnaire encyclopédique du théâtre. Paris. Bordas,1995, p. 119). Il s'agit de révéler des situations d'oppression (d'où le terme générique de "théâtre de l'opprimé»). A travers la technique du «théâtre-forum », l'oppresseur et l'opprimé sont mis en scène, et «(...) le spectateur participe à l'action dramatique en pleine connaissance de cause » (à la différence de la technique du " théâtre invisible »). A. Boal. Jeux pour acteurs et non acteurs : pratique du théâtre de l'opprimé. Paris, La Découverte. 1995, p. 38.

32. Le meneur de jeu « (...) encourage ou invite toute volonté à s'exprimer sur scène (...). [il] propose une synthèse mais ne juge pas. I) fait le lien avec le réel et stimule d'autres réactions, d'autres improvisations. » extrait plaquette prestataire.

33. Extrait plaquette prestataire.

34. "Le théâtre interactif comme image de médiation au sens large, est un lieu de dialogue, de questionnements, de paroles, d'essais et d'affirmations. ». extrait plaquette prestataire

35. Affirmer que l'on pourrait grâce à des mises en scène spécifiques changer les mentalités, infléchir les comportements et donc influer sur les motivations, s'inscrit plutôt dans une perspective fonctionnaliste. humainement réductrice. A moins que ce ne soit jouer sur des ressorts plus manipulatoires mais dans ce cas, le spectateur n'est jamais véritablement dupe et se souvient avoir été dupé. Adopter une démarche volontaire ne suffit pas à imposer sa volonté. Maurice Blanchot dans L'espace littéraire (Paris, Gallimard. 1978. p. 268 et 347), a écrit que l'œuvre de l'écrivain s'accomplit dans l'écart de la lecture. Cet "écart » fait que son œuvre achevée lui échappe et l'en dessaisit. Nous pensons qu'il en est de même pour un spectacle.

36. Loin de nous l'idée de détourner les règles de construction de la dramaturgie classique (l'académique règle des trois unités de construction d'une pièce : unités d'action, de temps et de lieu)! 
37. «Le sens, pour l'anthropologue, c'est toujours le sens social, les significations instituées et symbolisées du rapport à autrui. ». Marc Augé. Pour une anthropologie des inondes contemporains. Paris, Flammarion, 1997. p. 22

38. E. Morin, Amour, poésie, sagesse. Paris. Editions du Seuil. 1999. p. 45.

39. R. Sainsaulieu, Sociologie de la culture : organisation, culture et développement. Ed. rev. et augm., Paris. Presse de la fondation nationale des sciences politiques \& Dalloz. 1997. p. 84.

40. Une relation : " un lien et un récit ». nous dit Patrick Baudry, La place des morts : enjeux et rites, Paris, Armand Colin. 1999, p. 197-198.

41. «Nous sommes en présence à travers communication et projet de deux processus humains qui employés à doses homéopathiques apparaissent comme des processus émancipateurs au regard des conditions existentielles des individus et des groupes : en favorisant le lien social, en développant la capacité à devenir acteur à travers ses décisions, ces deux processus sont effectivement porteurs de nouvelles formes de socialité. Leur usage intensif, voire intempestif lié à une forte demande culturelle qui s'est transformée sous nos yeux en effet de mode a vite amené des distorsions porteuses de déficits, voire de dérives pathologiques. Ce sont de telles distorsions à identifier et à conjurer, qui constituent aujourd'hui les défis auxquels nous nous trouvons confrontés. Répondre à ces défis, c'est retrouver sous projet et communication le rôle émancipateur qu'ils n'auraient jamais dû perdre.». J.P. Boutinet, « Management par projet et logique communicationnelle. quelles convergences, quels défis?». Communication \& Organisation $\mathrm{n}$ - $13.1^{\mathrm{er}}$ semestre 1998 , p. 220-221.

42. Nous pouvons nous demander si un positionnement trop "managérialiste" ne contribue pas à diluer la valeur créative du spectacle et celle, humaine, de la rencontre. Comme le rappelle JeanFrançois Chanlat, le "managérialisme" fait référence au «(...) système de description, d'explication et d'interprétation du monde à partir des catégories de la gestion (...), le produit d'une société de gestionnaires qui cherchent à rationaliser toutes les sphères de la vie sociale. ", J.F. Chanlat, Sciences sociales et management : plaidoyer pour une anthropologie générale, ParisMontréal, Eska-Les Presses de l'Université de Laval, 1998, p. 20.

43. P. Baudry, ibid., p. 62.

44. Cf. Jean Maisonneuve. Les conduites rituelles. Paris. Puf. 1995. J. Maisonneuve parle plus précisément de « réassurance contre l'angoisse » et de « maîtrise du mouvant ».

45. Partiellement détournée sur la forme, cette idée d'« aménageur » est empruntée à Georges Balandier (Le détour: pouvoir et modernité. Paris, Fayard. 1985, p. 236). Situant l'architecte Le Corbusier dans le courant de l'«imaginaire aménageur», l'auteur dit de ce dernier «(...) il urbanise en accordant la priorité à la logique fonctionnelle et instrumentale au détriment de la logique symbolique.» Mais pour autant. G. Balandier rappelle que Le Corbusier a imposé sa marque sur le siècle et qu'il est créateur de l'espace de la modernité. Nous retiendrons essentiellement ici

46. Y. Winkin, Anthropologie de la communication. Bruxelles. De Boeck Université. 1996. p. 83.

47. Même si ces aspects peuvent présenter une certaine séduction.

48. Isabelle Orgogozo, Les paradoxes du management : des châteaux forts aux cloisons mobiles. Paris, Les Editions d'Organisation. 1991. p. 164.

49. Article intitulé «Le théâtre d'entreprise comme outil de management ». Courrier Cadres n 1241. daté du 20 février 1998. .

50. Cf. note de bas de page $n^{\circ} 50$

51. C. Castoriadis. op. cit.. p. 109

52. Jean-Paul Fischer et Jean-Baptiste Perret. «La mimesis sociale: l'approche historique de Gunter Gebauer et Christoph Wulf », Hermès n²2, p. 63

53. J. Maisonneuve, op. cit.

54. Exemple: pour "faire prendre conscience» à une secrétaire qu'elle laisse sonner le téléphone trop longtemps avant de répondre, on met en scène une situation humoristique qui 
stigmatise les déconvenues de cette attitude. Quelle considération de la personne émerge de ce choix ? La saynète est-elle relayée par une action de formation ou s'y substitue-t-elle?

55. A l'instar de l'analyse sur la question des médias et de la démocratie proposé par Georges Balandier dans son ouvrage Le Dédale, ne pourrait-on pas considérer ce qui peut advenir si « (...) le comédien se convertit en metteur en scène des idées ?(...) La relation ainsi établie est forte dans l'immédiat, vulnérable dans la durée; elle a la fragilité des images qui la façonnent: elle se trouve exposée au risque du désamour et de la versatilité, des variations rapides du crédit et du discrédit. ». G. Balandier, Le dédale : pour en finir avec le XX siècle. Paris, Fayard, 1994, p. 208.

56. Cf. Michel Crozier et Erhard Friedberg, L'acteur et le système. Paris, Editions du Seuil. 1977.

\section{RÉSUMÉS}

«Théâtre d'entreprise » est une expression dans l'air du temps, qui évoque communément du théâtre fait pour l'entreprise, voire par les acteurs de l'organisation. Après en avoir rappelé l'émergence, cet article met en avant l'hétérogénéité des pratiques disséminées et la multiplicité des acteurs concernés. Les modalités et les enjeux de ces approches théâtrales dans les organisations diffèrent selon les intentions de l'offre et de la demande. Les dimensions communicationnelles de ces formes théâtrales et la nature des relations qu'elles présupposent sont envisagées. On peut également caractériser un théâtre d'entreprise, de nature plus managériale, et en souligner les arguments. Le discours ainsi analysé oscille entre des aspects qualifiés d'émancipateurs et d'aménageurs.

This article deals with « Corporate Drama ». As a matter of fact « Corporate Drama »means plays made for an organization, indeed even played by employees. In most cases, plays aim at motivating. This article analyses the specificities of these techniques in the field of communication. The emphasis is laid on the communication process.

\section{INDEX}

Mots-clés : théâtre, stratégie, management, technique, intervention, représentation, culture, imaginaire

\section{AUTEUR}

\section{ISABELLE COUSSERAND}

Isabelle Cousserand est allocataire de recherche en sciences de l'information et de la communication. Elle prépare une thèse de doctoral consacrée aux dimensions de la mémoire en communication des organisations. Elle est également monitrice d'enseignement supérieur au sein de la filière Communication d'Entreprise à l'I.U.T. Michel de Montaigne-Bordeaux 3. 